\title{
Energy Efficient Data Transmission Control using Adaptive Duty Cycle Terminology in a Real Time Sensor Application
}

\author{
Pugalvadivu.S ${ }^{1}$, Haripriya. $R^{2}$, Sowmiya. $A^{3}$, Abirami. $P^{4}$ \\ PG scholar, Department of Electronics and Communication Engineering, School of Communication and \\ Computer Science, Kongu Engineering College, Perundurai, Erode, Tamil Nadu, India ${ }^{1,2,3,4}$
}

\begin{abstract}
Energy saving and Congestion avoidance are the crucial issues in wireless sensor networks. In existing paper consider mostly how to reduce energy without data information, importance and timing issue. In this paper to overcome this issue by effective utilization of adaptive duty cycle terminology in Energy Efficient Data Transmission Control (EEDTC) algorithm in a real time application. The data transmission is control by using different duty cycle task (RTOS task-Real Time Operating System Task) for different data types. Duty cycle control for Real time application is a crucial issue because of timing problem. This is adaptively achieved using priority based data transmission in wireless sensor networks.
\end{abstract}

Keywords: Wireless sensor network, Duty cycle, RTOS task, Scheduling, Congestion, EEDTC

\section{INTRODUCTION}

Nowadays, Internet is the motherland of technology. In (temperature, vibration, pressure). The multi-hop topology that, wireless sensor networks(WSN) is the highest network increases energy efficiency in communication. integrated new generation of wireless network, which Various applications of sensor networks are habitat has high potential and wide range of applications such as monitoring, tracking chemical plumes and smart improving the existing scheduling, presenting new transportation. The intelligent sensor network is done by scheduling algorithm, increasing network lifetime, effective utilization of duty cycle (WAKEUP-time, commercial, industrial, military and space research areas. SLEEP-time).This is done by three type of scheduling It consists of prolific applications in the various domains algorithms named as synchronous, asynchronous and which includes cell phone monitoring, robotic exploration, hybrid.

disaster management, intrusion detection and medical systems. In the recent years, researchers have been trying to improve the efficiency of these networks. A thorough survey of this algorithm is important for the outlook to understand the current research and to determine the future innovative works in this domain.

Inclusion in wireless networking as micro-fabrication, integration of embedded microprocessors lead to new generation of adaptive scale sensor network for military and commercial applications. Wireless sensor network platform includes wireless motes or modules (microcontroller, memory, communication), sensing board (sensing devices) and programming work board (connect to network). General architecture of WSN is defined in the fig.1. The main processes of wireless sensor networks are sensing, processing and communicating to real world. Unlike central control system, a sensor network has unique set of resource constraints such as limited on-board battery power and limited communication bandwidth. Typical sensor network has microcontroller and small amount of memory for signal processing and task scheduling. Each sensor also has one or more devices such as acoustic microphone array, infrared, videos or still cameras, seismic, or magnetic sensor. Sensor network integrates internet deep into the physical environment. The resulting network is more expansive and dynamic than the current TCP/IP network. Information collection and transmission on sensor network express the condition of physical world
Synchronous based nodes know each other. The node latency is demonstrated by four components. 1) Sent time: This time is expressed by the sender to construct the message, including delays by operating system calls context switching and data to the network interface. 2) Access time: Collision is incurred while waiting for access of transmission channel due to collision, congestion and contention. 3) Propagation time: This is the delay incurred while the message travels across the channel to destination node (for single-hop wireless transmission it is negligible whereas in multi-hop it is increased). 4) Receive time: This is the time when the receiver node transmits the data to the network interface. For e.g. consider two nodes as q, w. Node $\mathrm{q}$ reads clock with time $\mathrm{t} 1$ and sends packet to node $\mathrm{w}$. While receiving the packet at node $\mathrm{w}$, it records time as $t 2=(t 1+D+d)$, where $D$ is unknown delay in the channel between two nodes, $d$ is phase difference. Due to the presence of clock skew, frequent resynchronization may be required, whereas in asynchronous scheduling method, data is transmitted without considering whether other nodes are awake to receive. It doesn't require any computation as well as communication and storage overhead, since no scheduling are exchanged and stored. Its challenge is how to transmit data between two nodes which are not aware of each other's wakeup. They have 1)transmitter initiated 2)receiver initiated 3) Random or combination of both of them. They use special frame overhead. Asynchronous method is used in light load 
condition. Final one is hybrid scheduling method MAC protocol layer for the energy consumption of WSN. (Combination of synchronous asynchronous(method).Compared to global synchronization, pair-wise synchronization is easier. Hybrid has moderate computational complexity but major drawback is, it requires large storage and communication overhead. This is because, addition of message was exchanged and stored to enable nodes to ascertain the proper mode of operation to use. The main contributions of sensor networks are faster data transmission and enhancement of network life time. Energy waste is created by idle listening, overhearing (listening of unwanted frame), control packet overhead, collision and congestion. While attempting to reduce idle listening, it leads collision problem, and it results in control traffic process. So every action is linked with one another. When selecting the microprocessor for sensor network, we need to consider the energy consumption level of the processor. Active radio consumes energy as twice of active CPU.Global time synchronous at rendezvous (strict synchronous) scheme is advantageous, because of its high capability to coordinate

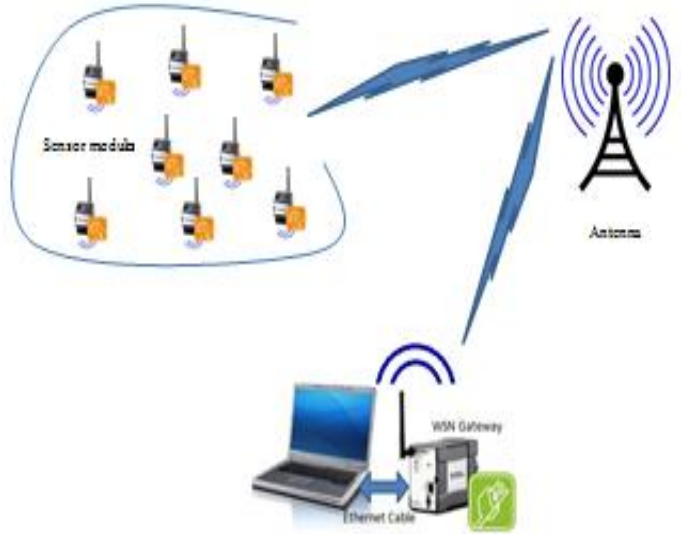

Fig. 1. Wireless Sensor Network Architecture

transmission, reducing idle listening and collision at the same time. The remainder of this paper is organized as follows. Existing related work are presented in section II. Design assumption and duty cycle parameter are discussed in section III. Proposed work are explained in section IV. Result analysis is compared in section V. Finally concluded with future work in section VI.

\section{RELATED WORK}

The entire existing surveyed model has a common objective of maximizing sensor network lifetime. They create different assumption based on sensors application. It takes fair comparison with existing surveyed mechanism Based on Duty cycle, the classification is done as follows ( Fig. 2).

WSN functions [10][5] leads into four different states such as, Initialization, Wake-Up, Collection, and Inactive, based on this information, it dynamically changes its sleep and wake up time. [4] [6] Increase of network life time is done by using mostly off duty cycle algorithm. This kind of algorithm create latency and congestion problem. [7] Based on node density the sink queries are sensed by sensing components of each sensor. [8]AS2-MAC is new

\section{[9][11] SMAC having fixed ON and OFF time.[12]The}

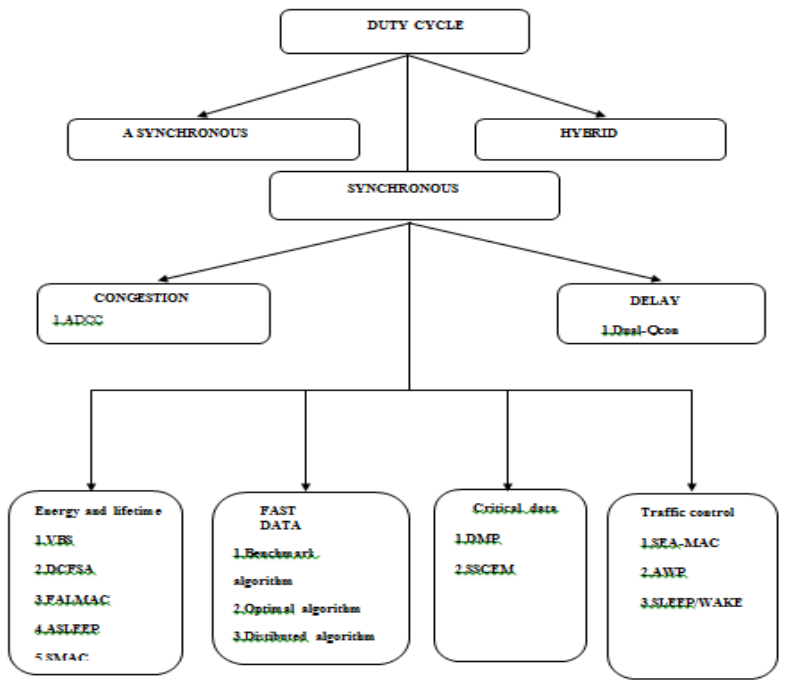

Fig. 2. Duty cycle classification

increase of sensor density creates congestion problem, that results in low throughput and high packet energy consumption, so it leads to degrade performance in WSN. Generally congestion can be alleviated by 1 .Traffic control and 2.Resource control. Fast data transmission[13] they use three types of algorithms, non-duty cycle case and based on insight of general case, optimal algorithm and distributed algorithm are used. Delay constraints [14] Dual-QCon controller design algorithm taken an account such as queue management and duty cycle control for end server. [15][16] Sleep/wake scheduling scheme is taken based on traffic condition. Nodes adopt scheduling scheme based on three factors such as the distance from sink node to another node, specifying the node location, whether the node is nearer to the event places. [17] The Adaptable Wakeup Period (AWP) focuses the receiving operation. Based on the traffic condition around a one hop distance, they adjust the active time at the receiver side. By the use of Clear Channel Assessment (CCA) value the traffic amount is measured. [18] Dynamic Multilevel Priority Packet Scheduling(DMP) has three type of queues. One is for high priority queues for real time data packet access. Another two queues are for non- real time data packet access. One is for remote data reception and another one is for local data reception. [20] [19]Sleep Scheduling for Critical Event Monitoring reduce the delay.

\section{PRELIMINARIES}

In this section, we explained general design assumptions and define some terminologies such as energy saving parameters that are used in designing the Energy Efficient Data Transmission Control(EEDTC) algorithm scheme.

\section{A. Energy saving parameter on sensor}

1) ON-DUTY: All activities of sensor part are turned on. This node is able to sense and collect the data from 
physical environment then process the data, and finally communicate to other node or gateway only when called in Wake-up period or Active period. This is not an energy saving part.

2) SENSING ON-DUTY: Processor and sensing part only turned on. The other part such as transceiver is turned off. Data could not be transmitted in this period but data processing is taken out.

3) TRANSCEIVER ON-DUTY: In this mode, processor and transceiver are turned on, but sensing unit is turned off. The data transmission and reception are carried out.

4) OFF-DUTY: In this part, whole communication, process and processors are turned off, but some other triggering mechanism may run to activate the sensor module. It is called as sleep state.

\section{B. Design assumptions}

- Synchronous duty cycle scheduling. Based on destination information the source node is controlled.

- $\quad$ Real time data based

- All priority data packets are same size

- Priority based data transmission

- Sensor nodes are located in different location from the gateway.

- $\quad$ Gateway is queue based scheduling

- $\quad$ Preemptive-Speed of response is high, and nonpreemptive based RTOS - speed of Response is low.

- $\quad$ Gateway queue monitoring

C. Duty Cycle calculation

\section{$>$ WAKE TIME}

\section{Wake up time \\ Total duration of sleep and wake up \\ $>$ SLEEP TIME}

\section{Sleep time \\ Total duration of sleep and wake up}

Duty cycle is a total duration of an ON and OFF time of data transmission timeslot. In this based on our application data priority the RTOS task time is varied. Here we used three different duty cycle task for three different data.

\section{PROPOSED WORK: ENERGY EFFICIENT DATA TRANSMISSION CONTROL(EEDTC)}

We have designed one new algorithm as adaptive duty cycle terminology in Energy Efficient Data Transmission Control(EEDTC) algorithm. The proposed strategy functionality are described by architecture diagram, it shown in fig. 3. We have three different task such as RTOS task 1, task 2, task 3. Based on application requirement condition the task selection changed. Here task 1 used for critical time(high priority) conditions, task 2 called by non-critical data(medium priority), and task 3 used for periodic data transmission. Each task had different duty cycle value.

$\rightarrow$ ON time of duty cycle

$\rightarrow$ OFF time of duty cycle

$\rightarrow$ queue Level of gateway

$\rightarrow$ Sensor value in queue memory

$\rightarrow$ Transceiver

$\mathrm{T}_{\mathrm{X}} \mathrm{R}_{\mathrm{X}}$

Critical data transmission

If(sensor value $>=$ high priority data)

\{

Immediately send the data

$\mathrm{T}_{\mathrm{X}} \mathrm{R}_{\mathrm{X}}=\mathrm{ON}$;

Duty cycle task 1 ;

Tox $=$ Transmission is $\mathrm{ON}$; $\quad$ /Wake up time for

Jensor

$T_{\text {OFs }}=$ Transmission is OFF; $\quad$ / Nap time (sleep)

for sensor

Non-critical data transmission

Else if(Medium priority data $\leqslant=$ Sensor value $s$ high priority data)

\{

Immediately send the data

$\mathrm{T}_{\mathrm{X}} \mathrm{R}_{\mathrm{X}}=\mathrm{ON}$;

Duty cycle task 2;

Tox $=$ Transmission is $\mathrm{ON}$; /Wake up time for sensor

$T_{\text {orst }}=$ Transmission is OFF; // Nap time (sleep) for sensor

Periodic data transmission

$\mathrm{Els}$

$\mathrm{T}_{\mathrm{X}} \mathrm{R}_{\mathrm{X}}=\mathrm{ON}$

Duty cycle task 3 ;

Tox = Transmission is ON; /Wake up time for sensor

$T_{\text {OrE }}=$ Transmission is OFF; // Nap time (sleep) for sensor

A. sensor node

The sensor node module is a source of data, this have controlled based on real time requirements. We take a three type of data with three different task schedules. Here we finished this algorithm by using real time hardware module with iSenses tool. Resource of sensor values are compared with our required value. If the sensing value is the greater than the our threshold value(critical value), then it will use a task 1 to time slot. This had a low duty 
cycle task. So the response of application is high. During critical data time, the running task could not stop their execution, because of non-pre-emptive method. After that execution it can changed their task. Now this has a preemptive task. If the sensing value is less than the noncritical data (medium priority) then, we use a task 2. Likewise task 1 also used. Here nap time is a sleep time of sensor node. In existing they used size based, FCFS-First Come First Served, EDF- Earliest Deadline First technique. But the main priority of data is missing.

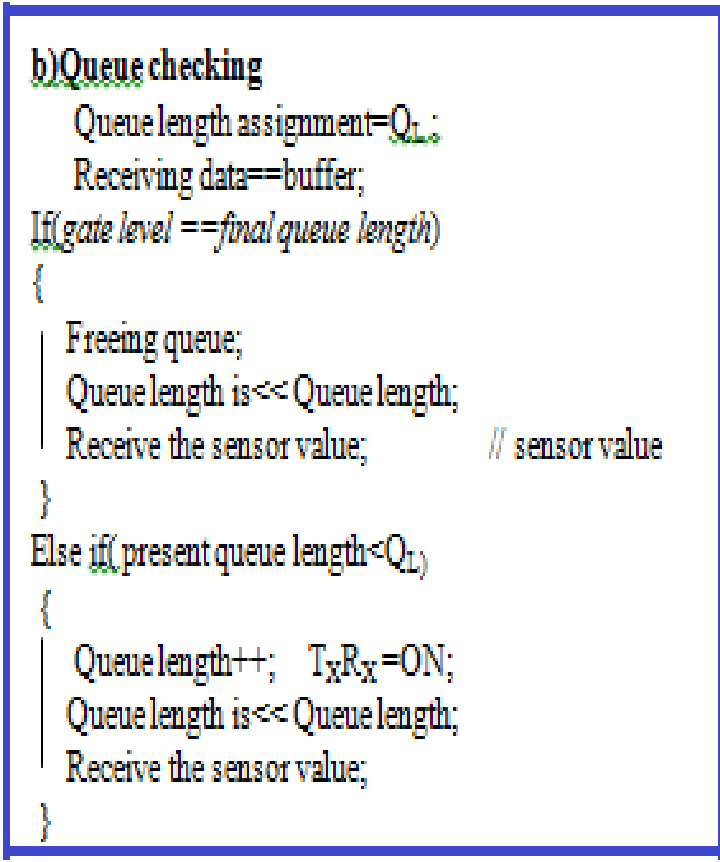

\section{B. Destination side}

Destination is a gateway node, which has a receiving data from various nodes. Every data has received at destination side. So the congestion problem is created. To minimize or clear this problem by using queue management with resource control. Based on our application we have changed the queue size. We have taken a queue size as 15 . If queue size is reached a $15^{\text {th }}$ level, then we freed the queue. Again queue is started.

\section{RESULT ANALYSIS}

Result analysis is taken by the real time hardware module with temperature sensor. Based on the proposed algorithm EEDTC the result are plot between time and transmission data, congestion, duty cycle. The hardware ishell output generator gave the output response for different task. Table.I show the WSN constraints values. The fig. 4 shows an data transmission time for various data type. During low duty cycle time only the transmission is increased, at awake time 1 second, the transmission value is high. During 10 second awake time the transmission is minimized because of high congestion at fig. 4. So the data transmission time increased with energy package are saved. At the time of 1 second awake slot create 3 second congestion, it showed in fig. 7, fig. 4,fig. 5,fig. 6. But during 5 second awake time the congestion value is 15

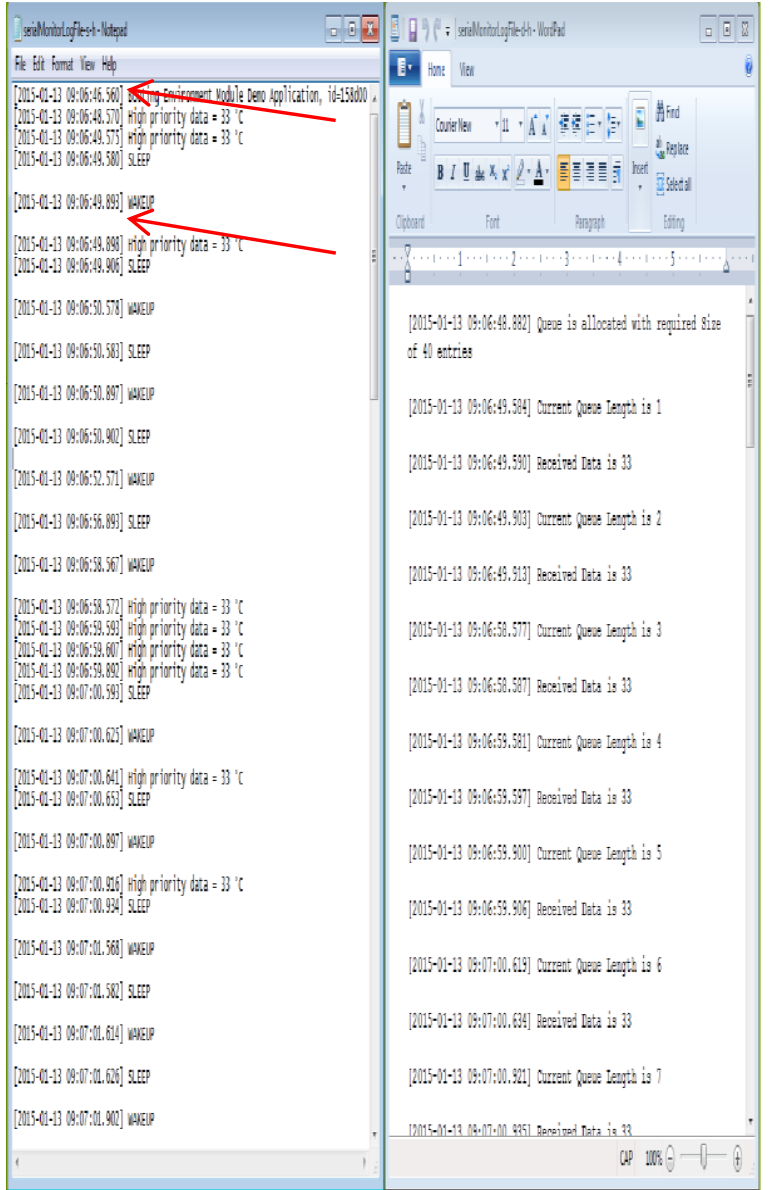

Fig. 4. 1Second Awake-3sec sleep

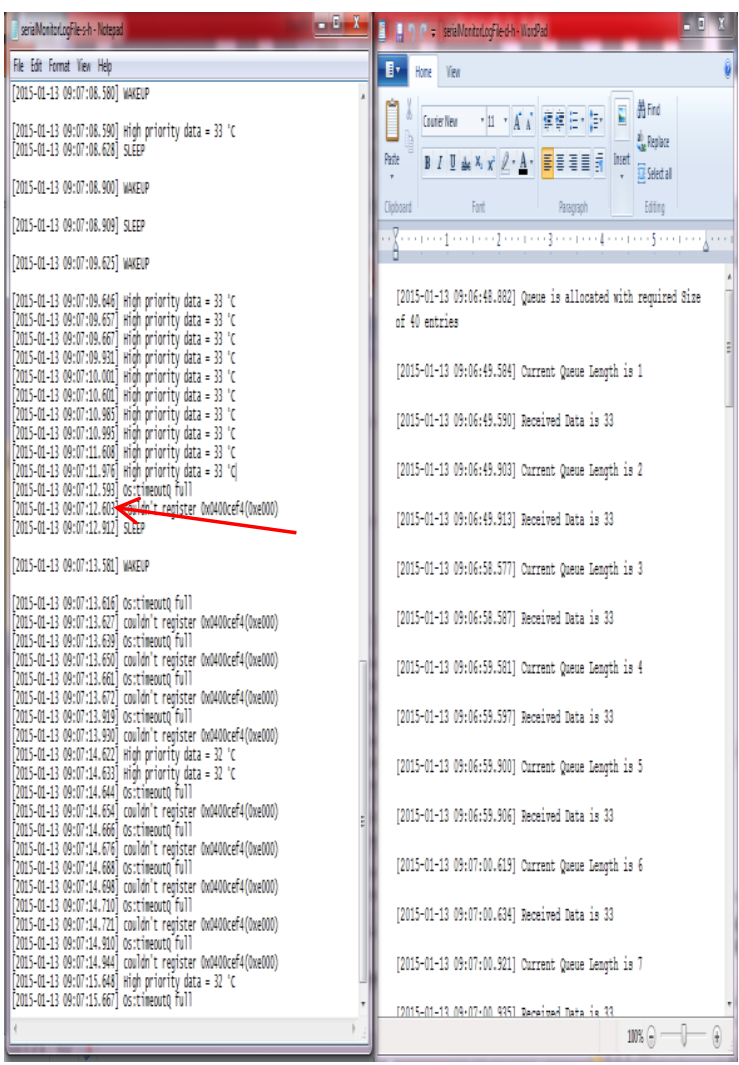

Fig. 5. 23 Second -Transmission 
because of fixed ON and OFF time. So from this graph we got an output as for different duty cycle task. Compare to 5 second awake the 10 second have low transmission time, because of high sleep time. Fixed data transmission duty cycle task have data loss, this analysis gave a critical data loss. So this not suitable for critical condition. Small application only used. But in the duty cycle scheduling with data partitioning at source side for congestion avoidance is showed a better result compare to fixed duty cycle data transmission such as SMAC. Destination Congestion linearly decreased .

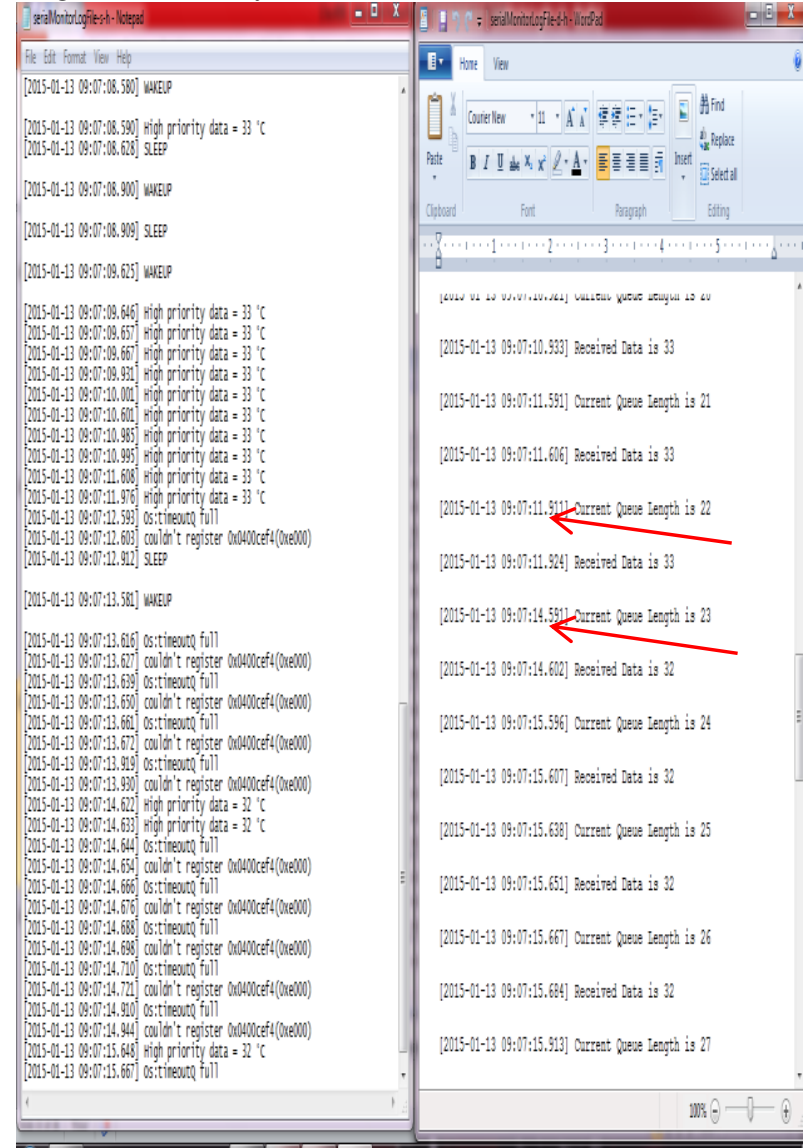

Fig. 6. 3 Second -Congestion

TABLE I

RESPONSE OF WSN CONSTRAINTS BASED ON DIFFERENT DUTY CYCLE

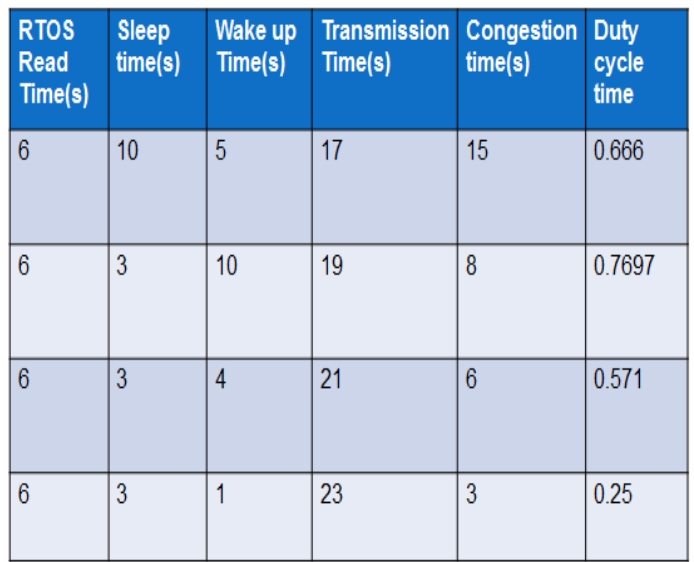

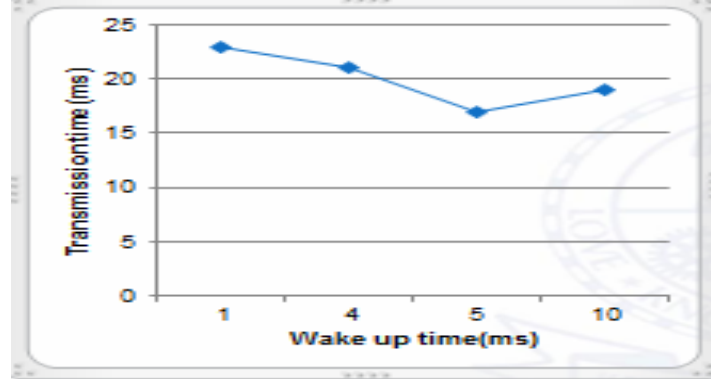

Fig. 7. Transmission time for various data

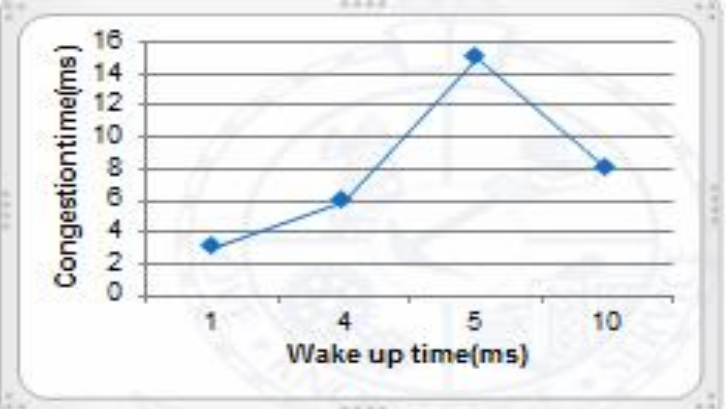

Fig. 8. Congestion for various data

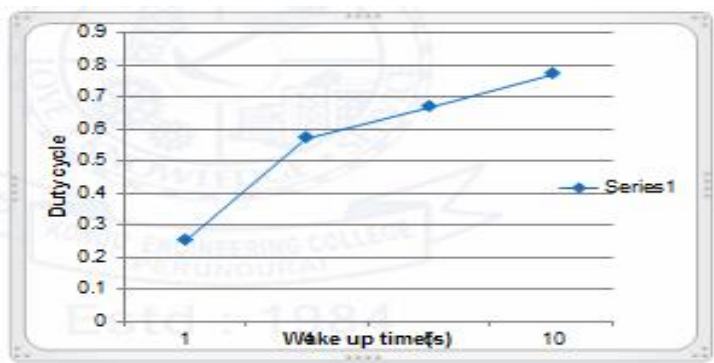

Fig. 9. Duty cycle for various data

\section{CONCLUSION}

In the previous scheduling which considers mostly on Energy conservation, topology, coverage, lifetime configuration, latency, connectivity, multi-queue under the categories of synchronous, asynchronous and hybrid. Also those algorithms are suitable for specific applications. Among them, most of the algorithms are based on effective utilization of energy. But the main aim of Wireless Sensor Networks is to increase data transmission rate with critical data indication. The EEDTC algorithm give better result. But the congestion is created because of different duty cycle task. This will be avoided in future by queue management and source side adaptive sleep time changes by pre-emptive scheduling algorithm.

\section{ACKNOWLEDGMENT}

We sincerely thank Self Organized Networking Group (SONG) and its members for their valuable comments and continuous support in conducting the survey on sensor network duty cycle scheduling. We also thank the unknown reviewers for their valuable suggestion and comments.

\section{REFERENCES}

[1] Yaxiong Zhao, JieWu,Fellow, Feng Li, and Sanglu Lu, “ On Maximizing the Lifetime of Wireless Sensor Networks Using Virtual Backbone Scheduling," IEEE Trans . parallel distributed systems, Vol. 23, No. 8, pp.1528-1535, Aug 2012. 


\section{International Journal of Advanced Research in Computer and Communication Engineering} Vol. 4, Issue 2, February 2015

[2] Gaurav S. Kasbekar, Yigal Bejerano, and Saswati Sarkar, "Lifetime and Coverage Guarantees Through Distributed Coordinate-Free Sensor Activation," IEEE/ACM Trans. networking, Vol. 19, No. 2, pp. 470-473,Apr 2011.

[3] Rashmi Ranjan Rout, and Soumya K. Ghosh, "Enhancement of Lifetime using Duty Cycle and Network Coding in Wireless Sensor Networks," IEEE Trans. wireless communications, Vol. 12, No. 2,pp.656-667, Feb 2013.

[4] Hady S. AbdelSalam, and Stephan Olariu, " Toward Adaptive Sleep Schedules for Balancing Energy Consumption in Wireless Sensor Networks," IEEE Trans. computers, Vol. 61, No. 10, pp.1443-1458, Oct 2012.

[5] Sudip Misra, Debashish Mohanta, " Adaptive listen for energyefficient medium access control in wireless sensor networks," Multimed Tools Appl, Springer Science ,pp- 121-145, Nov 2009.

[6] Giuseppe Anastasi, Marco Conti, and Mario Di Francesco, "Extending the Lifetime of Wireless Sensor Networks Through Adaptive Sleep," IEEE TRANSACTIONS ON INDUSTRIAL INFORMATICS, VOL. 5, NO. 3,pp. 351-351,Aug 2009.

[7] Yinying Yang, Mihaela Cardei, "Adaptive energy efficient sensor scheduling for wireless sensor networks," Springer- Verlag,vol 4, pp.359-369, July 2009.

[8] Luca Anchora, Antonio Capone, Vincenzo Mighali, Luigi Patrono, Francesco Simone, "novel MAC scheduler to minimize the energy consumption in a Wireless Sensor Network,"Ad Hoc Networks, Elsevier, vol 16, pp. 88-104, Dec 2013.

[9] Mehmet Yunus Donmeza, Sinan Isik, Cem Ersoy, "Combined analysis of contention window size and duty cycle for throughput and energy optimization in wireless sensor networks," Computer Networks ,Elsevier,vol 57,pp. 1101-1112, Dec 2012.

[10] Wojciech Bobera,b, Chris J. Bleakley, " BailighPulse: A low duty cycle data gathering protocol for mostly-off Wireless Sensor Networks," Computer Networks ,elsevier, vol 69, pp.51-65, May 2014.

[11] Wei Ye, John Heidemann, , and Deborah Estrin, Fellow, "Medium Access Control With Coordinated Adaptive Sleeping for Wireless Sensor Networks,"IEEE/ACM TRANSACTIONS ON NETWORKING, VOL. 12, NO. 3, pp.493- 506, Jun 2004.

[12] Lee and Kwangsue Chung, "Adaptive Duty-cycle Based Congestion Control for Home Automation Networks," IEEE Transactions on Consumer Electronics, Vol. 56, No. 1,pp.42-47, Feb 2010.

[13] Zhong Shen, Hai Jiang and Zhongjiang Yan,Member, "Fast Data Collection in Linear Duty-Cycled Wireless Sensor Networks ,'IEEE TRANSACTIONS ON VEHICULAR TECHNOLOGY, VOL. 63, NO. 4,pp. 1951-1957, May 2014.

[14] Heejung Byun, Soogook Son, Jungmin So, "Queue management based duty cycle control for end-to-end delay guarantees wireless sensor networks,"Springer Science+Business Media New York,Wireless Netw, vol 19 pp.1349-1360, Jan 2013.

[15] Babar Nazir, Halabi Hasbullah and Sajjad A Madani, "Sleep/wake scheduling scheme for minimizing end-to-end delay in multi-hop wireless sensor Networks," Journal on Wireless Communications and Networking, springer, pp.1-14, 2011.

[16] Y. Z. Zhao,C. Y. Miao, M. Ma, "An Energy-Efficient Self-Adaptive Duty Cycle MAC Protocol for Traffic- Dynamic Wireless Sensor Networks," Wireless Pers Commun, Springer, vol 68,pp.1287-1315, Jan 2012.

[17] Jae-Ho Lee, "A Traffic-Aware Energy Efficient Scheme for WSN Employing an Adaptable Wakeup Period," Wireless Pers Commun, Springer Science,vol 71, pp.1879-1914, Nov 2012.

[18] Nidal Nasser, Lutful Karim, and Tarik Taleb, "Dynamic Multilevel Priority Packet Scheduling Scheme for Wireless Sensor Network." IEEE TRANSACTIONS ON WIRELESS COMMUNICATIONS, VOL. 12, NO. 4, pp. 1448-1459,Apr 2013.

[19] Peng Guo, Tao Jiang, Qian Zhang, Fellow and Kui Zhang, “Sleep Scheduling for Critical Event Monitoring in Wireless Sensor Networks," IEEE TRANSACTIONS ON PARALLEL AND DISTRIBUTED SYSTEMS, VOL. 23, NO. 2, pp. 345-352, Feb 2012.

[20] Shibo He, Jiming Chen, Senior Member, and Youxian Sun, "Coverage and Connectivity in Duty-Cycled Wireless Sensor Networks for Event Monitoring, "IEEE TRANSACTIONS ON PARALLEL AND DISTRIBUTED SYSTEMS, VOL. 23, NO. 3, pp.475-482,Mar 2012.

[21] Messaoud Doudou, Djamel Djenouri, and Nadjib Badache, "Survey on Latency Issues of Asynchronous MAC Protocols in DelaySensitive Wireless Sensor Networks,"IEEE COMMUNICATIONS SURVEYS \& TUTORIALS,VOL.15, NO.2,pp. 528-550,2013.
[22] Ricardo C. Carrano, Diego Passos, Luiz C. S. Magalh aes, and C'elio V. N. Albuquerque, "Survey and Taxonomy of Duty Cycling Mechanisms in Wireless Sensor Networks,"IEEE COMMUNICATIONS SURVEYS \& TUTORIALS, VOL. 16, NO. 1, pp.181-194, 2014.

[23] www.coalesenses.com 